\title{
Wetting Resistance of Heterogeneous Superhydrophobic Coatings with Orthogonally Layered Fibers
}

\author{
T.M. Bucher, M.M. Amrei, and H. Vahedi Tafreshi* \\ Department of Mechanical and Nuclear Engineering, Virginia Commonwealth University, \\ Richmond, Virginia 23284-3015
}

\begin{abstract}
Superhydrophobic coatings comprised of electrospun nanofibers are a low-cost alternative to micro-fabricated surfaces, and can be applied to substrates of any arbitrary geometry. Such coatings with orthogonally oriented layers have properties that allow their wetting resistance to be predictable for a range of solid volume fractions, fiber diameters, and contact angles. In this paper, we have presented a modeling strategy that solves for the airwater interface shape over several layers of such coatings to predict the resistance of superhydrophobic fiber coatings to hydrostatic pressures and to quantify the relationship between microstructure, meniscus penetration depth, and wetted surface area of the fibers. Slip length predictions are also provided to shed some light on the performance of such coatings in drag reduction applications. It was found that while failure pressure for a coating rises with reducing fiber spacing, there is a tradeoff with wetted fiber surface area relative to a bare substrate. This tradeoff can be offset, however, by using smaller fibers for an intended coating. This results in a higher failure pressure for the same wetted area fraction. The results generated in this work are discussed in relation to those reported in the literature whenever possible.
\end{abstract}

Keywords: Fibrous coatings; superhydrophobicity; contact angles; dissimilar fibers; wettability

\footnotetext{
*Address correspondence to Hooman Vahedi Tafreshi, Department of Mechanical and Nuclear Engineering, Virginia Commonwealth University, email: htafreshi@ vcu.edu; tel.: 804-828-9936; fax: 804-827-7030;

URL: http://www.people.vcu.edu/ htafreshi/
} 


\section{Introduction}

A surface is regarded as superhydrophobic (SHP) when it exhibits an apparent contact angle with water greater than $150^{\circ}$. This is often achieved by applying a micro- or nano-scale roughness to a hydrophobic surface [1-4]. Such surfaces can be used for applications ranging from self-cleaning and drag-reduction to corrosion resistance and energy [4-6]. The essential attribute of SHP surfaces is their reduced water-solid contact area (wetted area) which helps decrease the friction between a moving body of water and the surface. This is due to the ability of SHP surfaces to trap air within their microstructure (see e.g., [7-10]). For an SHP surface in contact with water, the solid area in contact with water depends on both the surface morphology and the hydrostatic and/or hydrodynamic conditions of the system. A submerged SHP surface may be found to be in the Wenzel state (fully wetted), the Cassie state (fully dry), or in a series of transition states between the two extremes [9]. When an SHP surface comes into contact with water, the air-water interface (AWI) may penetrate into the pores of the surface depending on the wetting state of the surface. If the AWI penetrates too deeply into the pores, the SHP surface may no longer provide any drag reduction. In fact, it is quite possible that such a surface increases the drag force in certain hydrodynamic conditions [10].

A significant number of studies have been devoted to the manufacture and characterization of micro-fabricated roughness on a surface to impart superhydrophobicity (see e.g., [5]). However, fabricating micro- or nanoroughness remains a costly process, and applying them to geometries with arbitrary curvatures remains difficult. An alternative is to achieve the desired roughness by applying a hydrophobic material to the surface in the form of electrospun nanofibers [11-14] or apply a coating on the surface of a fibrous material [15-17]. The major problem with the conventional electrospinning process (or fibrous materials in general), however, is the lack of control over the orientation and spatial distribution of the fibers, making it difficult to predict the performance of the coating prior to its manufacturing. It has been shown that the conventional electrospinning process can be modified to produce coatings with some additional control over the orientation of the fibers and their spacing (e.g., [18-21]). Producing coatings with fiber layers arranged orthogonally with respect to one another similar to 
the one shown in Fig. 1 provides a means for engineering superhydrophobic coatings with more predictable performance. The example SEM image shown in this figure is from an electrospun superhydrophobic polystyrene mat with orthogonal fibers having an average fiber diameter of $2.4 \mu \mathrm{m}$ and a mat solid volume fraction (SVF) of 7.5\% [22-23]. Unlike coatings with random fiber orientations, orthogonally layered fiber mats have a degree of order to them that allows one to predict their overall performance based on an analysis conducted on a small portion of their structure, i.e., the spaces between the fibers conforming to a grid of rectangular cells. In this paper, we study the ability of orthogonally layered fibrous coatings to resist hydrostatic pressures. In particular, we obtain a critical pressure, the pressure at which a wetting transition can be expected, for coatings with different microstructures. This is accomplished by solving the surface energy equation across the AWI in the rectangular unit cells of the coatings to resolve the curvature of the interface to its minimum energy. These calculations are performed using the finite-element Surface Evolver (SE) code [24-25]. It is worth mentioning that the modeling methodology presented here can also be used in applications involving oil-water separation [26-30], water transport in fuel cells [31-34], waterproof barriers for underwater devices [35], and self-cleaning (e.g., [36-37]) among many others.

In the remainder of this paper, we will first establish the failure criteria with which we define critical pressure for a given coating (Section 2). We will then explain the details of constructing our model in Section 3, and also present a mesh-independence study to ensure that the numerical errors associated with our numerical calculations are negligible. In Section 4, we conduct a parameter study to determine the critical pressure of coatings with different fiber diameters and porosities, as well as contact angles. In addition, we will present an investigation on the effects of fiber size dissimilarity on the performance of SHP coatings. Finally, we state our conclusions in Section 5 . 


\section{Critical Capillary Pressure}

\subsection{Coatings Comprised of Highly Oriented Fibers}

In general, an SHP coating is regarded to have failed under hydrostatic pressures when the applied pressure is sufficiently high to overtake the capillary pressure of the coating. The only exception to this is the case of thin coatings with the air under the AWI completely trapped, like when a coating is fully submerged (in this case the entrapped air significantly contributes to the coating's resistance against the hydrostatic pressure [38-39]). For thick coatings (i.e., coatings for which the volume of the air displaced by a penetrating AWI is negligible compared to the volume of the air in the coating), even when submerged, the capillary pressure is the dominant force balancing the hydrostatic pressure exerted on the coating [40].

For fibrous coatings with fibers highly oriented in a certain direction, the capillary pressure can be derived from the balance of forces acting on the AWI between the fibers (see Fig. 2) [40],

$$
p_{F B}=-\frac{2 \sigma \sin (\theta+\alpha)}{l-d \sin \alpha}
$$

where the center-to-center distance $l$ is related to the coating's SVF $\varepsilon$,

$$
l=\frac{\pi d}{4 \varepsilon}
$$

The critical capillary pressure $p_{F B}^{c r}$ can then be obtained by differentiating Eq. 1 with respect to $\alpha$, setting it equal to zero. Thus, critical capillary pressure across a bank of parallel fibers, expressed in terms of SVF and critical immersion angle $\alpha^{c r}$, can be written as

$$
p_{F B}^{c r}=-\frac{2 \varepsilon}{d} \frac{\sigma \sin \left(\theta+\alpha^{c r}\right)}{\pi-4 \varepsilon \sin \alpha^{c r}}
$$

It is worth mentioning that Eq. 3 was successfully used in our previous study to produce a 2-D AWI tracking algorithm, and thereby predictive pressure-saturation correlations, for porous media comprised of unidirectional fibers [41]. 
As mentioned earlier, Eqs. 1 and 3 are obtained for when the AWI is only in contact with two parallel fibers (2-D geometries). For a 3-D fibrous coating composed of multiple layers of orthogonal fibers like the one shown in Fig. 1, the capillary resistance is stronger, and the solid-water contact area is different for each fiber-layer with which the AWI is in contact. This is compounded by another issue: for a single set of parallel fibers, complete wetting takes place when the AWI penetrates deep enough to reach a critical profile, at a critical immersion angle of $\alpha^{c r}$, where the balance of mechanical forces over the AWI is no longer maintained [40-41]. As will be shown later in Section 3, our study indicates that an AWI that straddles across two or more layers of orthogonal fibers most often does not reach such a mechanical breaking point before it deflects laterally so as to meet itself under the fibers (across the symmetry boundaries). At this point, the AWI would probably coalesce with itself and break away from the first layer of fibers, nullifying the SHP characteristics of the coating by submerging the first layer. In such conditions, the capillary pressure right before the AWI coalesces with itself is taken in this work as the critical pressure (see the next section).

We previously used the Full Morphology (FM) algorithm to predict the resistance of submerged SHP fibrous coatings under elevated hydrostatic pressures [40]. The underlying principle of the FM method is to relate the size of the largest sphere that can fit into the void space between the fibers at any point in the 3-D domain to a fictitious pressure obtained from the Young-Laplace equation written for a capillary tube [42-43]:

$$
r_{s p h}=\frac{2 \sigma \cos \theta}{p_{F M}}
$$

This method, while computationally fast and flexible especially for studying randomly arranged 3-D fiber networks, is nonetheless an approximation treating the irregular void space between the fibers as a collection of cylindrical pores. In other words, an AWI penetrating into an electrospun coating does not necessarily conform to a spherical shape with a radius obtained from the local inter-fiber distance inside the 3-D fibrous structure. For the case of coatings comprised of parallel fibers, the critical capillary pressure can simply be estimated based on the size of the sphere that fits between the two fibers according to the FM method: 


$$
p_{F M}^{c r}=\frac{4 \sigma \cos \theta}{l-d}
$$

In a previous paper, we compared the predictions of Eqs. 3 and 5 for the simple case of two parallel fibers, and established a range of contact angles and fiber spacing where the two approaches closely agree [40].

\subsection{Coatings Comprised of Orthogonal Fibers}

For geometries more complicated than the special case of highly oriented fibers (e.g., fibrous coatings with random fiber orientations or orthogonal layers), no analytical expressions have yet been proposed for critical capillary pressure prediction. Except for the FM simulations of ref. [40], the closest attempt in predicting the critical pressure of a fibrous SHP coating with random fiber orientations is the work of ref. [39] in which a 2-D projection of the 3-D geometry of a thin fibrous coating was considered as the solution domain for solving an integro-partial differential equation (IPDE) to estimate the coating's critical pressure. Obviously, using the projected image of a 3-D structure is only accurate if the coating is very thin. Additionally, in the work reported in [39] the AWI was assumed to be pinned to the fibers (the inevitable consequence of using a 2-D solution domain). Nevertheless, despite the simplifications, the work of ref. [39] was the first to compare the performance of coatings with random fiber orientations with their counterparts having orthogonal fibers. While simulating the exact shape of the AWI in 3-D fibrous coatings comprised of fibers with random orientations remains a challenge for the future, the current paper uses the interfacial energy equation to numerically determine the shape of the AWI (and so the critical capillary pressure) for coatings made up of fibers with orthogonal orientations. More specifically, the modeling methodology developed here allows the AWI to freely move around the circumference of the fibers in response to hydrostatic pressures, and therefore can provide highly accurate predictions for both the critical pressure and wetted area of fibrous SHP coatings. 


\section{Model Construction and Formulations}

While a fibrous coating generally consists of many layers, we only considered the first four layers of staggered fibers in our study. This is because, according to our preliminary calculations, for the given range of SVFs considered here, an AWI cannot penetrate into the coating more deeply than four layers before the surface transition to the Wenzel state. Figure 3a, represents a cell from a coating comprised of four orthogonally layered fibers. The fibers are color-coded by layer for illustration. Here, $l$ represents the center-to-center spacing between adjacent fibers in the same layer. The layers have a staggered pattern, in order to better characterize the spaces through which an AWI would penetrate into the coating. The domain has symmetry boundary conditions around the outer fibers (see Fig. 3b).

The SE code is able to solve for the minimum-energy shape of an interface between two immiscible fluids. The general form of the energy equation being integrated in the code can be expressed as [44]:

$$
E=p \iiint d V-\sum \sigma \cos \theta_{i} \iint d A_{i}
$$

where $p$ is the applied pressure difference across the interface. The summation refers to the energy contributed by the wetted area of each fiber associated with the interface. To ensure proper calculation of the fibers' energy contribution, the integrand $d A_{i}$ must be derived for each fiber and applied explicitly in the code. Figure $3 \mathrm{c}$ is a visualization of the appropriate wetted-area element for the fibers in any layer of a coating (the fiber is aligned in the $x$-direction). In our virtual coatings, the fibers in the first layer are always oriented in the $x$-direction. The positive $z$-direction is down into the coating with $z=0$ at the top. The AWI is symmetrically draped over the top of the fiber, pinned at angle $\alpha$ from the top of the fiber. The area element $d A$ for the fiber is thus derived as

$$
d A=d \alpha d x
$$

where through geometry

$$
\alpha=\cos ^{-1}(1-2 z / d)
$$


A similar modification must also be made in the code for the calculation of the volume integral in Eq. 6 . To better describe this, we show a cross-section of a fiber in the first layer in contact with an AWI intersecting at point $A$ (at an angle $\alpha$ from the top) in Fig. 3d. Without any correction, the contact line represented in 2-D by point $A$ would be projected in a straight line to the $x y$-plane, ignoring the overlapping fiber and the excess water above the fiber. To compensate for this, we write an additional volume integrand along the fiber. Two geometric regions are drawn with the fiber in Fig. 3d: an overlapping circular sector and trapezoid (regions I and II, respectively). Our volume integrand is now obtained by subtracting the area of region I, and adding the area of region II (area occupied by both regions I and II cancel). Using the convention of Eq. 8, we have

$$
d V=-\frac{1}{8} d^{2} \alpha d x+\frac{2 z+d}{8} d \sin \alpha d x
$$

Thus for a given pressure the SE code with customized area and volume elements (Eqs. 7 and 9) solves for the minimum-energy shape of the AWI, reporting back the volume, AWI depth, and wetted surface area of the fibers in contact with the water.

Figures $4 \mathrm{a}-4 \mathrm{e}$ show AWI examples obtained from our SE calculations for a coating with an SVF of $10 \%$ and a fiber diameter of $10 \mu \mathrm{m}$ at different pressures. The red and blue fibers have a Young-Laplace contact angle (YLCA) of $130^{\circ}$ and $100^{\circ}$, respectively. The initial setup has the AWI in contact only with fibers on first layer. As pressure increases the AWI penetrates deeper into the pore space between the fibers and wets fibers of the lower layers. As can be seen in Fig. 4e, for a given pressure of $4.60 \mathrm{kPa}$, the AWI has deflected sufficiently to come into contact with the fourth fiber layer without breaking off from the first layer. Figure $4 \mathrm{f}$ shows the same AWI but from a view that better illustrates the failure condition.

For a submerged body, as mentioned earlier, SHP surfaces can be used for skin-drag reduction purposes. The reduction in the skin-friction drag is often described by slip length [5]. An expression for slip length over SHP monofilament woven screens is suggested in ref. [36] as:

$$
b=\frac{L_{s}}{3 \pi} \ln \left(\frac{2\left(1+\sqrt{1-A_{w}}\right)}{\pi A_{w}}\right)
$$


where $A_{w}$ is dimensionless wetted area. Note that Eq. 10 was originally developed for planar structures, i.e., $A_{w}$ never exceeds 1 . Nonetheless, in the lack of a better alternative, we apply this formula as a means of studying the effects of pressure on slip length, but only as long as $A_{w} \leq 1$.

To ensure that the results presented here are not dependent on the choice of mesh-size considered for the numerical calculations, we considered a fibrous structure with an SVF of 10\%, a fiber diameter of $10 \mu \mathrm{m}$, and an YLCA of $120^{\circ}$. We considered uniformly distributed triangular elements with varying densities and monitored how mesh density $\lambda$ influenced the critical capillary pressure and wetted area. As it can be seen in Fig. 5, the simulations reach a state of mesh-independence at a mesh density of about $\frac{d}{\lambda}=10$. For the numerical results presented in this paper, a mesh density of $\frac{d}{\lambda}=10$ or greater was considered.

\section{Results and Discussion}

\subsection{Coatings with Unimodal Fiber Diameter Distributions}

Figure 6a shows critical capillary pressure and wetted area values for coatings comprised $10-\mu \mathrm{m}$ fibers and a YLCA of $120^{\circ}$. Note that $A_{w}=1$ is the surface area of the substrate as if the coatings were not applied. As shown graphically in the insets of Fig. 6a, at low SVFs the AWI is in contact with four fiber-layers at the moment of failure (when the AWI swells laterally to cross the symmetry boundary). At higher SVFs however, the failure occurs when the AWI is in contact with three fiber-layers only, and that is the reason why there is a slight change in the slope of the wetted in Fig. 6a for SVFs greater than about $10 \%$. Note in this figure that, critical pressure rises in a relatively linear fashion as SVF increases. This linear rise is in spite of the number of layers in contact with the AWI. It can be seen that, while a less porous SHP coating has a higher critical pressure for a given fiber size and YLCA, this effect comes at the expense of increased wetted area, i.e., potentially more friction with water. Figure $6 \mathrm{~b}$ shows the dimensionless slip length as a function of SVF for the same coatings. It can be seen 
slip length decreases with increasing the coating's SVF. As expected, slip length is smaller for coatings with larger wetted area. Note that Eq. 10 is only valid for when $A_{w}<1$.

Figure 6c shows the critical pressure versus SVF for coatings with square unit cells consisting of fibers with an YLCA of $120^{\circ}$ and diameters ranging from 5 to $15 \mu \mathrm{m}$. As can be seen in the figure, the critical pressure increases with increasing the SVF of the coating, as expected, and the relationship is almost linear. Figure 6c also shows an inverse linear relationship between fiber diameter and critical pressure (e.g., coatings with fibers three times smaller have a critical pressure three times higher for the same SVF). This relationship is consistent with that given analytically in Eqs. 3 and 5 (critical pressure scales with the inverse of fiber diameter at a constant SVF):

$$
\frac{p_{2}^{c r}}{p_{1}^{c r}}=\frac{d_{1}}{d_{2}}
$$

Therefore, one can easily extend the critical capillary pressures obtained for a coating with a fiber diameter of 10 $\mu \mathrm{m}$ to other coatings with different fiber diameters. Note that wetted area is independent of fiber diameter, and since critical pressure is observed to vary linearly with fiber diameter, a higher desired critical pressure can be achieved by using smaller fibers when the SVF is kept constant.

It is worth mentioning that it is convenient to display our results in terms of the SVF of the coatings, as opposed to more scale-specific metrics such as fiber spacing or fiber count. The SVF property, derived in relation to fiber spacing in Eq. 2, and the observed trends that accompany it are consistent across all length scales, whereas other more experimentally convenient systems of measurement are not. If fiber count per unit length $f_{c}$ happened to be the preferred metric, it is simply the inverse of fiber spacing $l$-and so the inverse of Eq. 2 (see e.g., ref. [45]).

As mentioned earlier, there are no analytical expressions to predict the critical pressure of SHP fibrous coatings beyond those having highly oriented fibers. Representing the 3-D structure of a pair of orthogonal fiber-layers with an unrealistic square-shape opening (i.e., treating the two orthogonal layers as one layer of interpenetrating fibers), Litster et al. [31] suggested an expression for the critical pressure of their fictitious media as follows: 


$$
p_{L i t}^{c r}=\frac{2 \sigma \cos \theta}{l-d}
$$

Equation 12 is in fact a modified form of the Young-Laplace equation. Predictions of Eqs. 5, 12, and 3 are compared with the results of our simulations in Fig. 6d for a coating with an SVF of 10\%, fiber diameter of 10 $\mu \mathrm{m}$, and an YLCA of $120^{\circ}$. This was done for both the case of a pore size accounting for only the first two layers, and a pore size accounting for the first four layers (red shaded regions in Fig. 6d). As is shown in this figure, Eqs. 3 and 5 agree well with one another for both modeled pores, and agree with our SE simulations of the coating $p^{c r}=4.8 \mathrm{kPa}$. Eq. 12 does not agree with any other data for any assumed pore size. It is important to note that, while the FM method (Eq. 5) agrees well with the FB equation (Eq. 3) and our SE simulations for the given set of properties, it was established in ref. [40] that this agreement is heavily dependent on the choice of YLCA, with the neighborhood of $120^{\circ}$ being the only area with close agreement.

In the absence of experimental data for critical pressure and slip length of superhydrophobic fibrous coatings with orthogonal fibers, here we only compare our numerical simulations with the measurements of Ref. [36] which were conducted for a series of monofilament woven screens enhanced with an SHP coating. Table 1 reports the experimental data of ref. [36] along with our numerical simulations results obtained for orthogonal fibrous coating with matching fiber diameter and spacing. Despite the obvious geometric differences between our fibrous coatings and the woven screens and also the inaccuracies involved in using the slip length equation for such surfaces, the reasonable agreement between the computational and experimental data is appreciable. One should also recognize the approximate nature of critical pressure measurements conducted by vertically immersing a mesh into water (ref. [36]), as different parts of the mesh would be exposed to different hydrostatic pressures at different depths.

We also studied coatings comprised of fiber-layers with different YLCAs (e.g., coating made up of two different polymers) and also tested the effects of alternating the order of these layers in the coatings. Figure 7a shows the effect of YLCA on critical pressure when the SVF and fiber diameter are kept at $10 \%$ and $10 \mu \mathrm{m}$, respectively. It 
can be observed that critical pressure increases with YLCA while the wetted area decreases. The insets in Fig. 7a show examples of the critical AWI in such coatings with the red and blue fibers having YLCAs of $100^{\circ}$ and $130^{\circ}$ respectively. It can be seen that the AWI for the more hydrophobic structure has a tighter curvature than the other, corresponding to its higher critical pressure. The increased hydrophobicity of the blue coating is also observed to result in a lower wetted surface area, whereas more of the AWI is draped over the fibers of the red structure. Figure $7 \mathrm{~b}$ shows dimensionless slip length at the critical pressure for the same coatings as a function of YLCA. Unlike wetted area, dimensionless slip length seems to increase with YLCA. Note in Fig. 7b that dimensionless slip length becomes negative when the wetted area is too large. Figures $7 \mathrm{c}$ and $7 \mathrm{~d}$ show the critical pressure, wetted area, and dimensionless slip length versus SVF, alternating which of the two fiber types is in the even- or odd-numbered layers (see the red and blue fibers in the inset of Fig. 7c). It can be seen that coatings with the more hydrophobic fibers on top have higher critical pressure and wetted area and consequently lower slip length. Interestingly however, wetted area (and slip length) for coatings with SVFs greater than about $12.5 \%$ is independent of the order by which the fiber-layers are stacked. This is again due to the fact that, at the moment of failure, the AWI over a high-SVF coating is in contact with three layers of fibers as opposed to four layers. Comparing the data points for an SVF of $10 \%$ to that of Fig. 6 and 7a shows that introducing multiple hydrophobicities results in an overall lower critical pressure and higher wetted area (lower slip length) than if only the more hydrophobic fiber type were used to produce the coating.

\subsection{Coatings with Bimodal Fiber Diameter Distributions}

Here, we examine the performance of orthogonally laid SHP fibrous coatings when alternating layers are comprised of fibers with different diameters. Figure 8a shows an AWI example in which fiber layers alternate in size for each layer. For such coatings, SVF must be defined over two layers (one fine fiber layer and one coarse). The ordered nature of such structures allows one to observe that the center-to-center spacing between the coarse 
and fine fibers $l_{c}$ and $l_{f}$ in a unit cell can be related to their corresponding number densities in the whole coating as to $l_{f} / l_{c}=n_{c} / n_{f}$. For our bimodal coatings, we define SVF as

$$
\varepsilon=\frac{\pi}{4 l_{f} l_{c}} \frac{d_{c}^{2} l_{f}+d_{f}^{2} l_{c}}{d_{f}+d_{c}}
$$

The coarse and fine fiber spacing $l_{c}$ and $l_{f}$ can be determined from Eq.13 to be

$$
\begin{aligned}
& l_{c}=\frac{\pi}{4 \varepsilon n_{c}} \frac{n_{c} d_{c}^{2}+n_{f} d_{f}^{2}}{d_{c}+d_{f}} \\
& l_{f}=\frac{\pi}{4 \varepsilon n_{f}} \frac{n_{c} d_{c}^{2}+n_{f} d_{f}^{2}}{d_{c}+d_{f}}
\end{aligned}
$$

where $n_{c}$ and $n_{f}$ are respective number fractions for coarse and fine fibers (e.g., for $n_{c}=0.1$, $10 \%$ of all fibers in an area of the coating are coarse fibers). It may also be convenient to express component fiber makeup in terms of coarse and fine mass fraction $m_{c}$ and $m_{f}$. These can be calculated from geometry as [46]:

$$
\begin{aligned}
& m_{c}=\frac{d_{c}^{2} l_{f}}{d_{c}^{2} l_{f}+d_{f}^{2} l_{c}}=\frac{n_{c} d_{c}^{2}}{n_{c} d_{c}^{2}+n_{f} d_{f}^{2}} \\
& m_{f}=\frac{d_{f}^{2} l_{c}}{d_{f}^{2} l_{c}+d_{c}^{2} l_{f}}=\frac{n_{f} d_{f}^{2}}{n_{c} d_{c}^{2}+n_{f} d_{f}^{2}}
\end{aligned}
$$

The results reported in this section are in terms of $n_{c}$, and like the cases mentioned in Sec. 4.1, there are trends that apply beyond the specific dimensions used in this paper. More specifically, it has been observed in our simulations that there is a range of $n_{c}(0.2$ or lower) in which critical pressure can be predicted analytically (i.e., without numerical simulations) via Eq. 3. This is because the AWI only comes into contact with the first two layers, and the domain size ratio $l_{f} / l_{c}$ is sufficient to allow the influence of the second layer to be neglected, hence the validity of Eq. 3. 
Figures $8 \mathrm{~b}$ and $8 \mathrm{c}$ present the critical pressure versus SVF and contact angle, respectively, for bimodal coatings comprised of fibers with diameters 10 and $50 \mu \mathrm{m}$ at an SVF of $10 \%$. The coarse fiber number fraction is $n_{c}=0.1$, and as can be seen in the inset figure, the aspect ratio of the unit cell is so large that the coating can actually be treated as a bank of parallel fibers with a diameter equal to that of the fine fibers $(10 \mu \mathrm{m})$. Perfect agreement between our simulations and the FB analytical equation (Eq. 3) is evident. The trend predicted by the FM method, which was previously reported in ref. [40], is also shown for comparison. In Fig. 8c, one can observe that FM method deviates from the two other methods for its purely geometric basis. In fact, the agreement between the predictions of Eq. 3 and the SE simulations is expected for any bimodal coatings in which the AWI does not come into contact with the third layer (wets only fibers on the first and second layers). Therefore, Eq. 3 and the geometry laid out in the inset of Fig. $8 \mathrm{~b}$ can be used to analytically determine whether or not this condition applies to a given coating. This is done by calculating the depth of the critical AWI $h^{c r}$ between two parallel fibers as:

$$
h^{c r}=h_{1}+h_{2}=\left(r_{f}+r_{f} \cos \left(\pi-\alpha^{c r}\right)\right)+\left(\sigma / p^{c r}-\sqrt{\left(\sigma / p^{c r}\right)^{2}-\left(l_{f} / 2-r_{f} \sin \alpha^{c r}\right)^{2}}\right)
$$

In this equation, $h_{1}$ is the depth of the immersion point $A$ measured from the top of the fiber and $h_{2}$ is the AWI maximum depth below the immersion point. If $h^{c r}$ is less than $d_{c}+d_{f}$ then Eq. 3 can be used to predict the critical pressure of the coating. Otherwise, numerical simulations such as those presented in this paper are needed. It is worth mentioning that for coatings with the top layer made of coarse fibers, the AWI most often comes into contact with three layers of fibers before failure occurs (unless when $n_{c}$ is very close to one), making it difficult to produce an analytical expression for predicting the critical pressure.

Figure 9a (fine fibers on top) and Fig. 9b (coarse fibers on top) show examples of the AWI over bimodal coatings with a coarse-to-fine fiber diameter ratio of $R_{c f}=4$ and different coarse fiber number fractions at the moment of failure. It can be seen in Fig. 9a that the AWI is in contact with two layers of fibers when $n_{c}=0.1$ and 0.2 , but comes into contact with the third and fourth layers for $n_{c} \geq 0.3$. For coatings with the coarse fibers on top (Fig. 
9b), the AWI remains in contact with three or four layers unless $n_{c}$ is very close to one (greater than at least $n_{c}=0.9$, not shown).

Figures 10a-10f show the critical pressure, wetted area, and slip length for bimodal coatings with different coarse fiber number fractions and coarse-to-fine fiber diameter ratios. Fine fiber diameter, SVF, and YLCA are all held at $10 \mu \mathrm{m}, 10 \%$, and $120^{\circ}$, respectively. Figs. $10 \mathrm{a}, 10 \mathrm{~b}$, and $10 \mathrm{c}$ present the results for when the first layer is made of fine fibers, and Figs. 10d, 10e, and 10f show the results with the coarse fibers on top. For better comparison between the critical pressure of bimodal and unimodal coatings, the critical pressure of the unimodal coating comprised of fibers with a diameter of $10 \mu \mathrm{m}$ and the same YLCA and SVF (i.e., $p^{c r} \cong 4.8 \mathrm{kPa}$ from Fig. 6) is shown in Fig. 10a and 10d with red dashed line. It can be seen that adding larger fibers (either by increasing $R_{c f}$ or $n_{c}$ ) to a coating comprised of finer fibers results in a decrease in the coatings resistance against elevated pressures. This conclusion is in agreement with our previous observation reported in [40]. Figures $10 \mathrm{~b}$ and $10 \mathrm{e}$ show the corresponding wetted area $A_{w}$ for each of the coatings shown in Fig. 10a and 10d (note that the area of the coating's unit cell (i.e., $l_{c} l_{f}$ ) increases with $n_{c}$ ). Recall that for a unimodal coating with an SVF of $10 \%$, fiber diameter of $10 \mu \mathrm{m}$, and an YLCA of $120^{\circ}$, a critical wetted area fraction of $A_{w} \cong 0.8$ was reported in Fig. 6. This value is shown in Figs. 10b and 10e for comparison. It can be seen that, adding larger fibers (either by increasing $R_{c f}$ or $n_{c}$ ) to a coating comprised of finer fibers affects the coatings' wetted area fraction in a somewhat more complicated manner. For coatings with the top layer comprised of finer fibers, the critical wetted area is significantly smaller at low coarse fiber number fractions but it increases with $n_{c}$ as can be seen in Fig. 10b. For coatings with the coarse fibers on top, the critical wetted area fraction generally decreases with $n_{c}$. Exceptions to this trend are coatings for which the AWI transitions from wetting three layers to wetting four layers when the coarse number fraction is increases (see the AWIs shown in Fig. 10b and the inset images in Fig. 10e). From these results, one can generally conclude that adding larger fibers to a unimodal coating comprised of small fibers can adversely affect the coating's critical pressure but improve its critical wetted area fraction if the coarse fibers are 
not added to the first layer. It is worth mentioning that while $h^{c r}$ decreases with increasing $R_{c f}\left(p^{c r}\right.$ scales predictably with overall fiber size scale Eq. 10), $A_{w}$ is fixed across all applicable scales. Thus, $p^{c r}$ can be raised by using smaller fibers, but $A_{w}$ will be preserved. The slip length results shown in Fig. 10c and Fig. 10f correspond to coatings with their first layer on top made of fine and coarse fibers, respectively. It can be seen that slip length is bigger at lower $n_{c}$ values for coatings with the top layer comprised of the finer fibers. On the other hand, for coatings with the coarse fibers on top, dimensionless slip length generally increases with $n_{c}$. As explained before, exceptions to these trends are when the AWI transitions from wetting three layers to wetting four layers, as the coarse number fraction is increased.

On a final note, calling back to Section 4.1, while SVF is useful for reporting the trends in this paper across a broad size scale, it may not be a practical basis for coating characterization experimentally. However, establishing fiber spacing $l_{f}$ and $l_{c}$ or fiber count per distance in the first few layers is more attainable via microscopy. Local or statistical SVF for a bimodal coating can then be calculated using Eq. 12. The number fractions $n_{c}$ and $n_{f}$ can then be determined via Eqs. 13a and 13b.

\section{Conclusions}

Our results show that the shape of the AWI that penetrates into the void space between the fibers of a coating are scalable with fiber diameter, making critical pressure from one fiber diameter to another equal to the inverse of the fibers' diameter ratio. This allows the trends illustrated in this paper to apply across the micro- or nano-scales. Our results also show the degree to which critical pressure improves with increasing SVF, but shows a limitation to this benefit due to wetted area of the coating becoming greater than the surface area of the substrate for higher SVFs, which causes significant slip length reduction. This tradeoff between critical pressure and wetted area (slip 
length) can be offset by introducing bimodality in fiber diameter over alternating layers, the finer of the two fiber sizes on top. This reduces critical pressure somewhat, but reduces wetted contact area to a greater degree. The critical pressure reduction can simply be addressed by using smaller fibers for the coating, as this would still preserve the reduced contact area. Finally, our results also show that, while critical pressure is higher and wetted area is lower for more hydrophobic fibers, alternating hydrophobicity from one layer to the next results in a somewhat lower critical pressure and somewhat higher wetted area than if only the more hydrophobic fibers were used. The effect of the order in which hydrophobicity is used in the layers of the coating is relatively minimal. Overall, bimodality in fiber size has a much more positive impact on the performance of orthogonal coatings.

\section{Acknowledgement}

The financial support from the National Science Foundation CMMI program grant number 1029924 is gratefully acknowledged. The authors also wish to acknowledge Dr. Ken Brakke for his help in coding portions of the SE model presented in this work, and Drs. Tepper and Ghochaghi of VCU School of Engineering for the SEM image shown in Fig. 1. 


\section{Nomenclature}

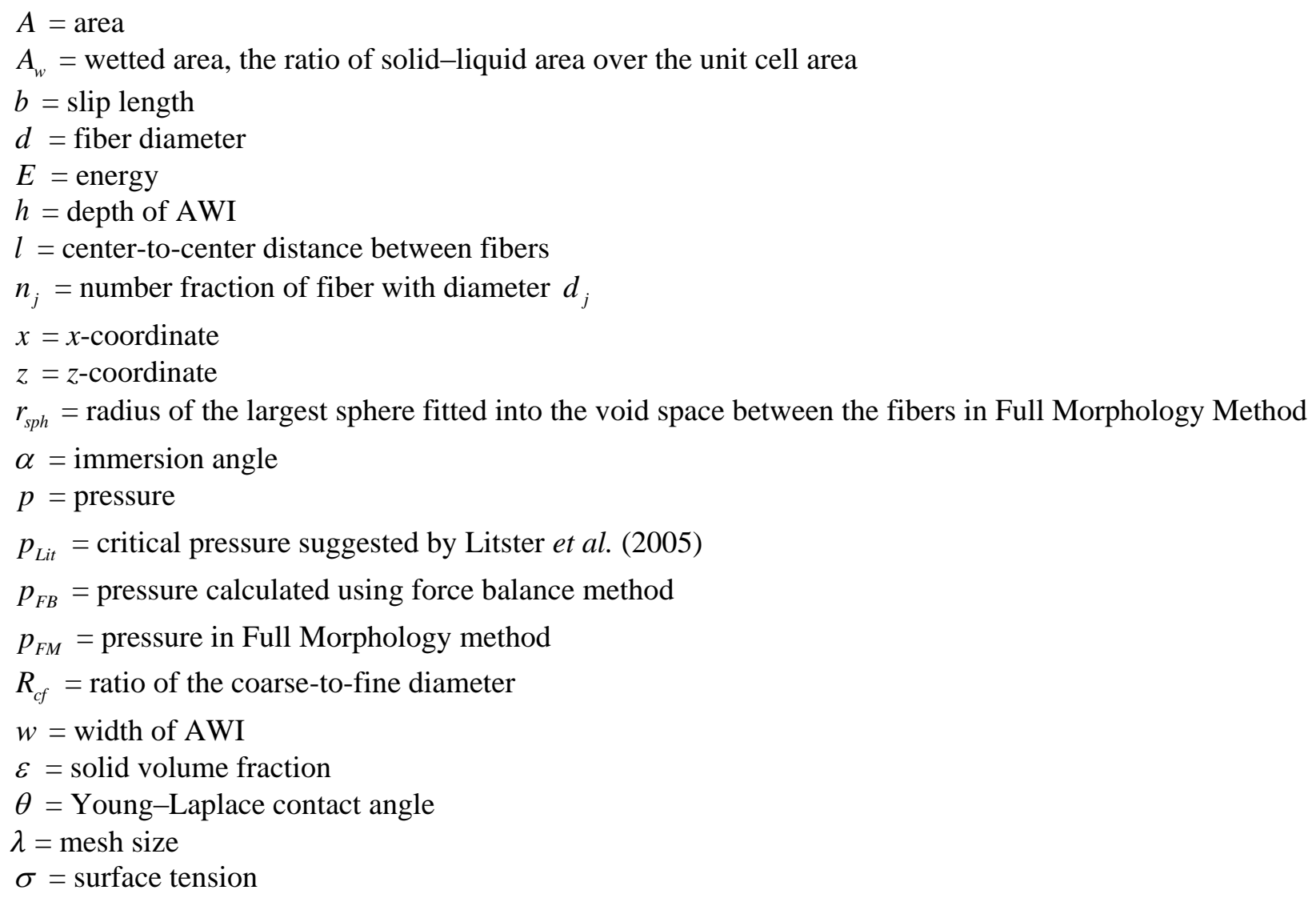

\section{Abbreviations:}

SE: Surface Evolver

FB: Force Balance

FM: Full Morphology

AWI: Air Water Interface

SVF: Solid Volume Fraction

YLCA: Young-Laplace Contact Angle

\section{Subscript:}

${ }_{c}=$ coarse fiber

$f=$ fine fiber

\section{Superscript:}

${ }^{c r}=$ the value correspondent to critical pressure 


\section{References}

1. A. Lafuma, D. Quere, Superhydrophobic states, Nat. Mater. 2 (2003) 457-460.

2. P. Roach, N.J. Shirtcliffe, M.I. Newton, Progress in superhydrophobic surface development, Soft Matter 4 (2008) 224-240.

3. N.J. Shirtcliffe, G. McHale, S. Atherton, M.I. Newton, An introduction to superhydrophobicity, Adv. Colloid Interface Sci. 161 (2010) 124-138.

4. D. Quere, Wetting and roughness, Ann. Rev. Mater. Res. 38 (2008) 71-99.

5. C. Lee, C.H. Choi, C.J. Kim, Structured surfaces for giant liquid slip, Phys. Rev. Lett. 101 (2008) 064501 .

6. R. Asmatulu, M. Ceylan, and N. Nuraje, Study of Superhydrophobic Electrospun Nanocomposite Fibers for Energy Systems, Langmuir 27 (2011) 504-507.

7. C.W. Extrand, Repellency of the lotus leaf: resistance to water intrusion under hydrostatic pressure, Langmuir 27 (2011) 6920-6925.

8. B. Emami, H.V. Tafreshi, M. Gad-el-Hak, G.C. Tepper, Simulation of meniscus stability in superhydrophobic granular surfaces under hydrostatic pressures, Colloid Surf. A-Physicochem. Eng. Asp. 385 (2011) 95-103.

9. M.M. Amrei and H.V. Tafreshi, Effects of hydrostatic pressure on wetted area of submerged superhydrophobic granular coatings. Part I: Monodisperse Coatings, Colloid Surf. A-Physicochem. Eng. Asp. 465 (2015) 87-98.

10. E. Karatay, A.S. Haase, C. W. Visser, C. Sun, D. Lohse, P. A. Tsai, R. G. H. Lammertink, Control of slippage with tunable bubble mattresses, Proc. Natl. Acad. Sci. U. S. A. 110 (2013) 8422-8426.

11. M.L. Ma, R.M. Hill and G.C. Rutledge, A review of recent results on superhydrophobic materials based on micro- and nanofibers, J. Adhes. Sci. Technol. 22 (2008) 1799-1817.

12. H. Wu, R. Zhang, Y. Sun, D. Lin, Z. Sun, W. Pan, and P. Downsb, Biomimetic nanofiber patterns with controlled wettabillity, Soft Matter 4 (2008) 2429-2433.

13. B. Shin, K.R. Lee, M.W. Moon, and H.Y. Kim, Extreme water repellency of nanostructured low-surfaceenergy non-woven fabrics, Soft Matter 8 (2012) 1817-1823.

14. N. Nuraje, W.S. Khan, Y. Lei, M. Ceylan and R. Asmatulu, Superhydrophobic Electrospun Nanofibers, J. Mater. Chem. A 1 (2013) 1929-1946.

15. C.T. Hsieh, F.L. Wu, and S.Y. Yang, Superhydrophobicity from composite nano/microstructures: Carbon fabrics coated with silica nanoparticles, Surf. Coat. Technol. 202 (2008) 6103-6108.

16. B. Xu, Z. Cai, W. Wang, and F. Ge, Preparation of superhydrophobic cotton fabrics based on $\mathrm{SiO} 2$ nanoparticles and $\mathrm{ZnO}$ nanorod arrays with subsequent hydrophobic modification, Surf. Coat. Technol. 204 (2010) 1556-1561.

17. Y. Zhao, Y. Tang, X. Wang, and T. Lin, Superhydrophobic cotton fabric fabricated by electrostatic assembly of silica nanoparticles and its remarkable buoyancy, Appl. Surf. Sci. 256 (2010) 6736-6742.

18. P. Katta, M. Alessandro, R.D. Ramsier, G.G. Chase, Continuous electrospinning of aligned polymer nanofibers onto a wire drum collector, Nano Lett. 4 (2004) 2215-2218.

19. S. Sarkar, S.C. Deevi, G.C. Tepper, Biased AC electrospinning of aligned polymer nanofibers, Macromol. Rapid Commun. 28 (2007) 1034-1039.

20. F.O. Ochanda, M.A. Samaha, H.V. Tafreshi, G.C. Tepper, and M. Gad-el-Hak, Fabrication of superhydrophobic coatings by DC-biased AC-electrospinning, J. Appl. Polym. Sci. 123 (2012) 11121119.

21. P.D. Dalton, C.V. Brooke, L. Farrugia, T.R. Dargaville, T.D. Brown and D.W. Hutmacher, Electrospinning and additive manufacturing: converging technologies, Biomater. Sci. 1 (2013) 171-185

22. N. Ghochaghi, PhD Dissertation, Department of Mechanical and Nuclear Engineering, Virginia Commonwealth University December 2014.

23. N. Ghochaghi, A. Taiwo, M. Winkel, B. Dodd, K. Mossi, and G. Tepper, Electrospun Polystyrene Coatings with Tunable Wettability, J. Appl. Polym. Sci. 132 (2015) 41592. 
24. K.A. Brakke, The Surface Evoler and the stability of liquid surfaces, Philos. Trans. R. Soc. A-Math. Phys. Eng. Sci 354 (1996) 2143-2157.

25. K.A. Brakke and F. Morgan, Instabilities of cylindrical bubble clusters, Eur. Phys. J. E 9 (2002) 453-460.

26. S.U. Patel and G.G. Chase, Separation of water droplets from water-in-diesel dispersion using superhydrophobic polypropylene fibrous membranes, Sep. Purif. Technol. 126 (2014) 62-68.

27. S.U. Patel, S.U. Patel, and G.G. Chase, Electrospun superhydrophobic poly(vinylidene fluoride-cohexafluoropropylene) fibrous membranes for the separation of dispersed water from ultralow sulfur diesel, Energy Fuels 27 (2013) 2458-2464.

28. S. Wang, M. Yu, J. Xue, W. Ou, A. Li, A superhydrophobic and superoleophilic miniature mesh box for oil spill cleanup, New J. Chem. 38(2014) 4388-4393.

29. D. Tian, X. Zhang, Y. Tian, Y. Wu, X. Wang, J. Zhai, L. Jiang, Photo-induced water-oil separation based on switchable superhydrophobicity-superhydrophilicity and underwater superoleophobicity of the aligned ZnO nanorod array-coated mesh films, J. Mater. Chem. 22 (2012) 19652-19657.

30. J. Song, S. Huang, Y. Lu, X. Bu, J.E. Mates, A. Ghosh, R. Ganguly, C.J. Carmalt, I.P. Parkin, W. Xu, C.M. Megaridis, Self-Driven One-Step Oil Removal from Oil Spill on Water via Selective-Wettability Steel Mesh, ACS Appl. Mater. Interfaces 6 (2014) 19858-19865.

31. S. Litster, D. Sinton, N. Djilali, Ex situ visualization of liquid water transport in PEM fuel cell gas diffusion layers, J. Power Sources 154 (2005) 95-105.

32. P. Zhou, C.W. Wu, Liquid water transport mechanism in the gas diffusion layer, J. Power Sources 195 (2010) 1408-1415.

33. L. Hao, P. Cheng, Lattice Boltzmann simulations of water transport in gas diffusion layer of a polymer electrolyte membrane fuel cell, J. Power Sources 195 (2010) 3870-3881.

34. D.A. Caulk, D.R. Baker, Modeling two-phase water transport in hydrophobic diffusion media for PEM fuel cells, Int. J. Electrochem. Soc. 158 (2011) B384-B393.

35. Y.M. Shin, S.K. Lee, J.Y. Lee, J.H. Kim, J.H. Park, C.H. Ji, Microfabricated environmental barrier using $\mathrm{ZnO}$ nanowire on metal mesh, J. Micromech. Microeng. 23 (2013) 127001.

36. S. Srinivasan, W. Choi, K.C. Park, S.S. Chhatre, R.E. Cohen, G.H. McKinley, Drag reduction for viscous laminar flow on spray-coated non-wetting surfaces, Soft Matter 9 (2013) 5691-5702.

37. S.T. Yohe, J.D. Freedman, E.J. Falde, Y.L. Colson, M.W. Grinstaff, A mechanistic study of wetting superhydrophobic porous 3d meshes, Adv. Funct. Mater. 23 (2013) 3628-3637.

38. B. Emami, H.V. Tafreshi, M. Gad-el-Hak, G.C. Tepper, Predicting shape stability of air-water interface on superhydrophobic surfaces comprised of pores with arbitrary shapes and depths, Appl. Phys. Lett. 100 (2012) 013104.

39. B. Emami, H.V. Tafreshi, M. Gad-el-Hak, G.C. Tepper, Effect of fiber orientation on shape and stability of air-water interface on submerged superhydrophobic electrospun thin coatings, J. Appl. Phys. 111 (2012) 064325.

40. T.M. Bucher, B. Emami, H.V. Tafreshi, M. Gad-el-Hak, G.C. Tepper, Resistance of nanofibrous superhydrophobic coatings to hydrostatic pressure: the role of microstructure, Phys. Fluids 24 (2012) 022109.

41. T.M. Bucher, H.V. Tafreshi, Modeling air-water interface in disordered fibrous media with heterogeneous wettability, Colloid Surf. A-Physicochem. Eng. Asp. 461 (2014) 323-335.

42. R.D. Hazlett, Simulation of capillary-dominated displacements in microtomographic images of reservoir rocks, Transp. Porous Media 20 (1995) 21-35.

43. M. Hilpert, C.T. Miller, Pore-morphology-based simulation of drainage in totally wetting porous media, Adv. Water Resour. 24 (2001) 243-255.

44. P.G. de Gennes, F. Brochard-Wyart, D. Quéré, Capillaryity and Wetting Phenomena: Drops, Bubbles, Pearls, Waves, Springer-Verlag, New York 2004.

45. T.M. Bucher, H.V. Tafreshi, G.C. Tepper, Modeling filtraion performance of nanofiber coatings with orthogonal fiber orientations, Powder Technol. 249 (2013) 43-53.

46. H.V. Tafreshi, M.S.A. Rahman, S. Jaganathan, Q. Wang, B. Pourdeyhimi, Analytical expressions for predicting permeability of bimodal fibrous porous media, Chem. Eng. Sci. 64 (2009) 1154-1159. 


\section{Figure Captions}

Figure 1: SEM image of an electrospun superhydrophobic Polystyrene mat with an average fiber diameter of 2.4 $\mu \mathrm{m}$ showing fiber-layers deposited orthogonally with respect to one another. The mat's average SVF and apparent water contact angle are about $7.5 \%$ and $140 \mathrm{deg}$., respectively.

Figure 2: Free body diagram of the balance of forces across the AWI between two parallel fibers.

Figure 3: (a) Visualization of a unit cell of an orthogonally layered fibrous coating comprised of four layers of fibers colored light blue, light red, dark blue, and dark red progressing into the coating. (b) Overhead view of the simulation domain. (c) Illustration of the energy contribution of a fiber in the first layer of a coating as water comes into contact with it. Wetted surface area as a function of $z$ is explicitly derived and coded into SE. (d) Illustration of the volume of a fiber in the first layer and additional water that must be accounted for in the model. Region I is deleted from the volume calculation. Region II is added. Volume as a function of $z$ is explicitly derived and coded into the model.

Figure 4: Sample simulation domain after refining mesh density and solving for the minimum energy shape and wetted area calculated by SE at different capillary pressures $(\mathrm{a}-\mathrm{e})$. The AWI is at its critical pressure when $p=4.6 \mathrm{kPa}$. This critical AWI is shown in (e) and (f) from two different viewpoints. Note that the AWI is approaching the symmetry boundary in (f).

Figure 5: Effects of mesh density on critical pressure and wetted area. The inset shows the mesh distribution for $\frac{d}{\lambda}=1.27$ and 9.09 .

Figure 6: (a) Critical pressure and wetted area fraction as a function of SVF for fibers with equal spacing on all layers. Top inset: AWI is four layers deep before meeting symmetry boundary. Bottom inset: AWI is only three layers deep before meeting symmetry boundary. (b) Dimensionless slip length as a function of SVF at the critical pressure for fibrous coatings with a fiber diameter of $10 \mu \mathrm{m}$ and an YLCA of $120^{\circ}$. (c) Critical pressure as a function of SVF for three different sets of coatings varying by fiber diameter. All coatings have a contact angle of $120^{\circ}$. (c) Red-shaded regions show overhead views of imaginary pores that one may assume to be formed by two or four layers of fibers in using Eqs. 3, 5, or 11. The critical pressure values obtained for the actual multi-layer coating with an SVF of $10 \%$, fiber diameter of $10 \mu \mathrm{m}$, and YLCA of $120^{\circ}$ using our SE simulations is $p^{c r}=4.8$ $\mathrm{kPa}$.

Figure 7: (a) Critical pressure and wetted area fraction and (b) dimensionless slip length as a function of contact angle for fibers with equal spacing on all layers. Coatings have an SVF of $10 \%$ and a fiber diameter of $10 \mu \mathrm{m}$. The red coating in the inset has a water contact angle of $100^{\circ}$, and the blue coating has a water contact angle is $130^{\circ}$.Sample image of a fibrous coating with alternating layer hydrophobicities at critical pressure. (c) Critical pressure as a function of SVF for coatings alternating in contact angle from one layer to the next. The alternating layers in the inset have contact angles of $130^{\circ}$ and $100^{\circ}$ for red and blue fibers, respectively. (d) Wetted area fraction and dimensionless slip length as a function of SVF for coatings alternating in contact angle from one layer to the.

Figure 8: (a) Sample domain for a coating with bimodal fiber diameter distribution. Structure has an SVF of $10 \%$, fine and coarse fiber diameters of 10 and $50 \mu \mathrm{m}$ respectively, and a coarse fiber number fraction $n_{c}$ of 0.4.Coating has an SVF of $10 \%$, fine and coarse fiber diameters of 10 and $50 \mu \mathrm{m}$ respectively, and a coarse fiber number fraction $n_{c}$ of 0.1 . (b) Critical pressure as a function of SVF. Simulation results are compared with analytical force balance relation and the Full Morphology method (Eqs. 3 and 5) from Bucher et al. [40]. Inset shows the geometry of the interface for calculating meniscus depth for Eq. 15. (c) Critical pressure as a function 
of contact angle for coatings. Simulation results are compared with analytical force balance relation and the Full Morphology method (Eqs. 3 and 5) from Bucher et al. [40]. Inset shows sample domain of a bimodal fibrous coating with an aspect ratio sufficient to facilitate the use of the force balance equation (3) across parallel fibers to predict critical pressure.

Figure 9: Meniscus configuration at critical pressure for various bimodal coatings varying in coarse fiber number fraction $n_{c}$. Coatings have an SVF of $10 \%$, fine and coarse fiber diameters of $10 \mu \mathrm{m}$ and $40 \mu \mathrm{m}$, and an YLCA of $120^{\circ}$.

Figure 10: Critical pressure, wetted area fraction, and slip length as a function of coarse fiber number fraction $n_{c}$ for bimodal fibrous coatings varying in fiber size ratio $R_{c f}$ from 2 to 5 are shown in (a),(b), and (c) for when fine fibers are on the top layer, and (d), (e), and (f) for when coarse fibers are on the top layer, respectively. Other properties shared by all coatings are shown in the figures.

Table 1: Comparison between the simulation data obtained for a four-layer fibrous coating and the experimental data of ref. [36] obtained for a woven monofilament screen with a matching contact angle of $124 \mathrm{deg}$. 

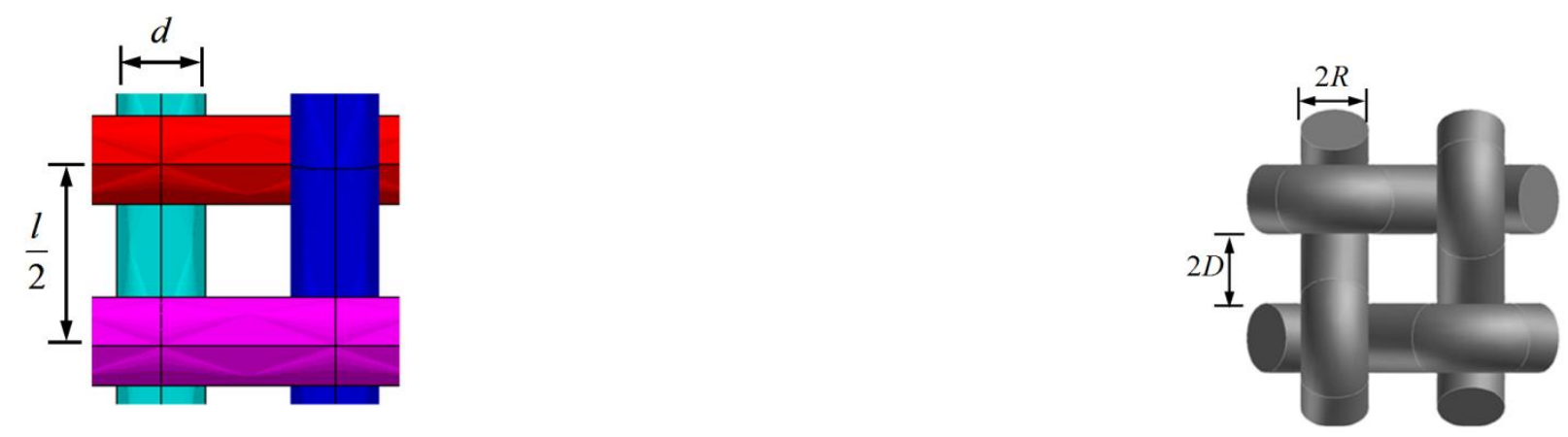

\section{Current study: fibrous coatings, four layers}

\section{Experiments of ref. [36]: woven mesh}

\begin{tabular}{|c|c|c|c|c|c|c|c|}
\hline$d(\mu \mathrm{m})$ & $l(\mu \mathrm{m})$ & Critical pressure $(\mathrm{Pa})$ & Slip length $(\mu \mathrm{m})$ & $R(\mu \mathrm{m})$ & $D(\mu \mathrm{m})$ & Critical pressure $(\mathrm{Pa})$ & Slip length $(\mu \mathrm{m})$ \\
\hline 140 & 598 & 310 & 63 & 70 & 229 & 190 & 82 to 102 \\
\hline 254 & 636 & 385 & 46 & 127 & 191 & 481 & 87 to 101 \\
\hline 254 & 906 & 195 & 79 & 127 & 326 & 180 & 143 to 171 \\
\hline 508 & 1412 & 125 & 86 & 254 & 452 & 112 & 170 to 218 \\
\hline 508 & 2114 & 80 & 220 & 254 & 805 & 56 & 198 to 228 \\
\hline 254 & 1694 & 110 & - & 127 & 720 & 28 & - \\
\hline
\end{tabular}

Table 1 
Figures
Click here to download Figure: Figures.pptx

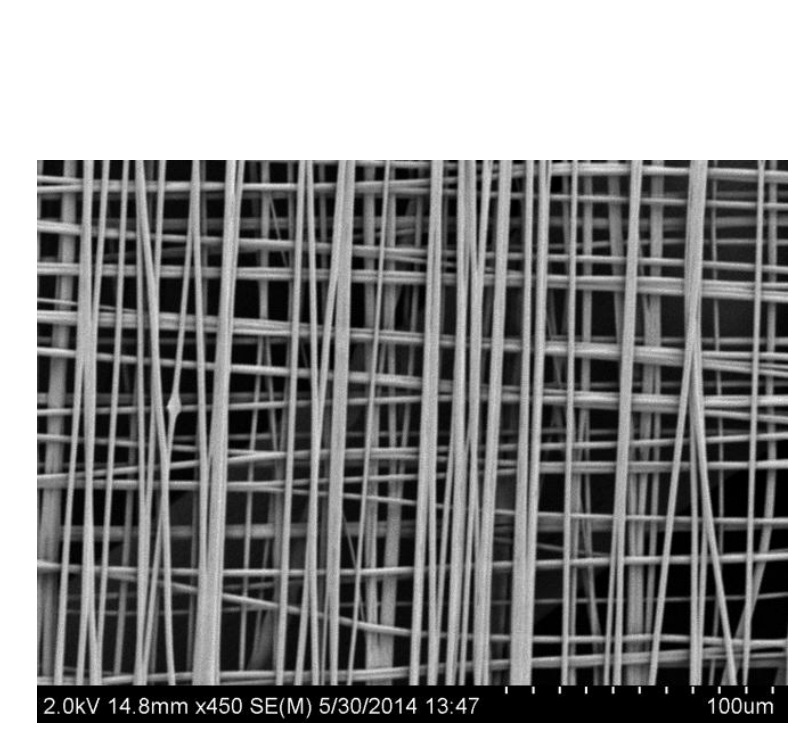

Figure 1

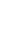

.

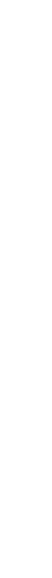




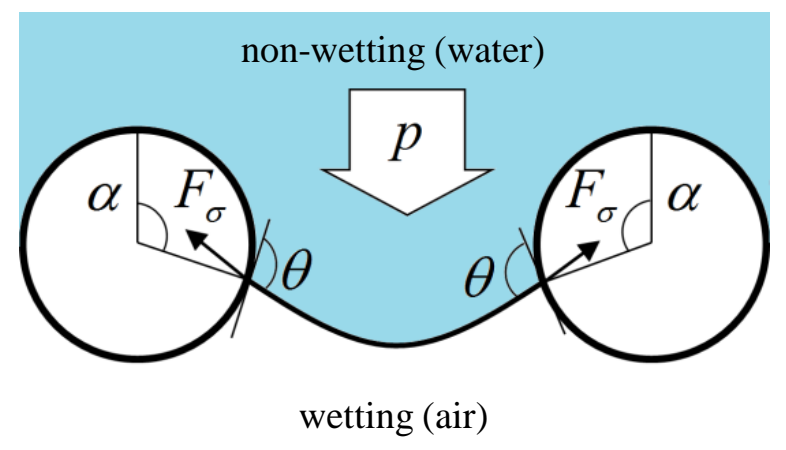

Figure 2 


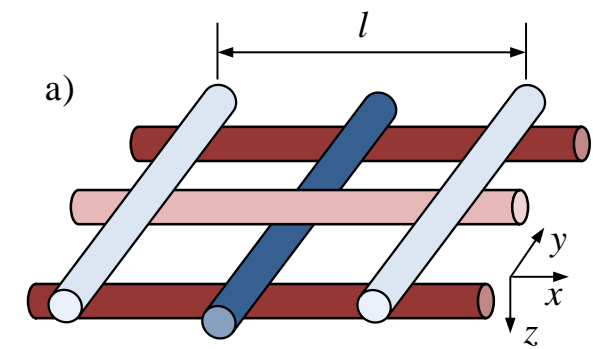

b) Symmetry
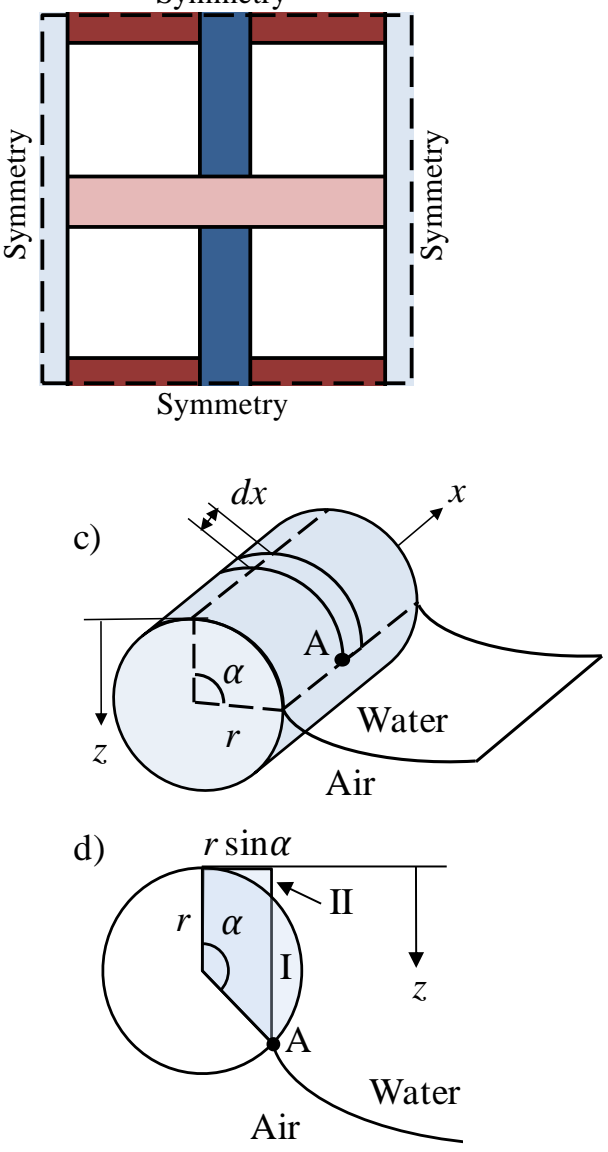

Figure 3 

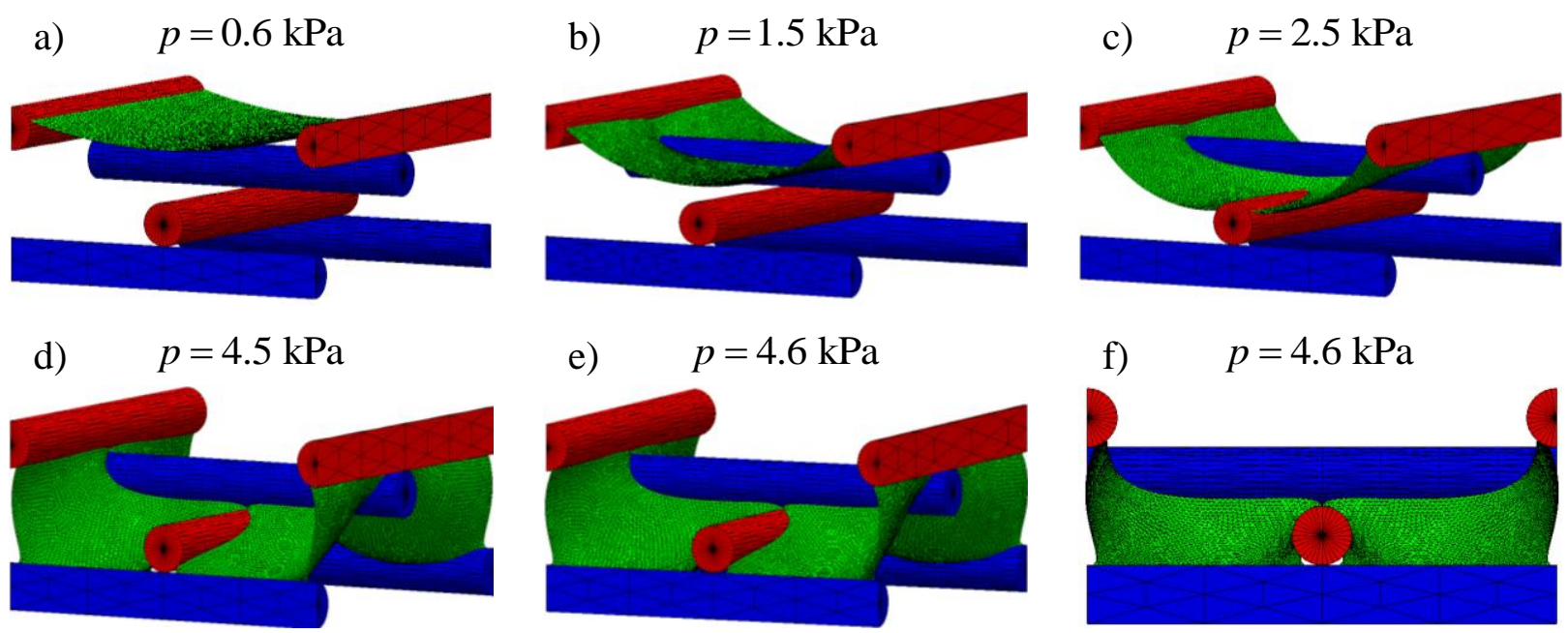


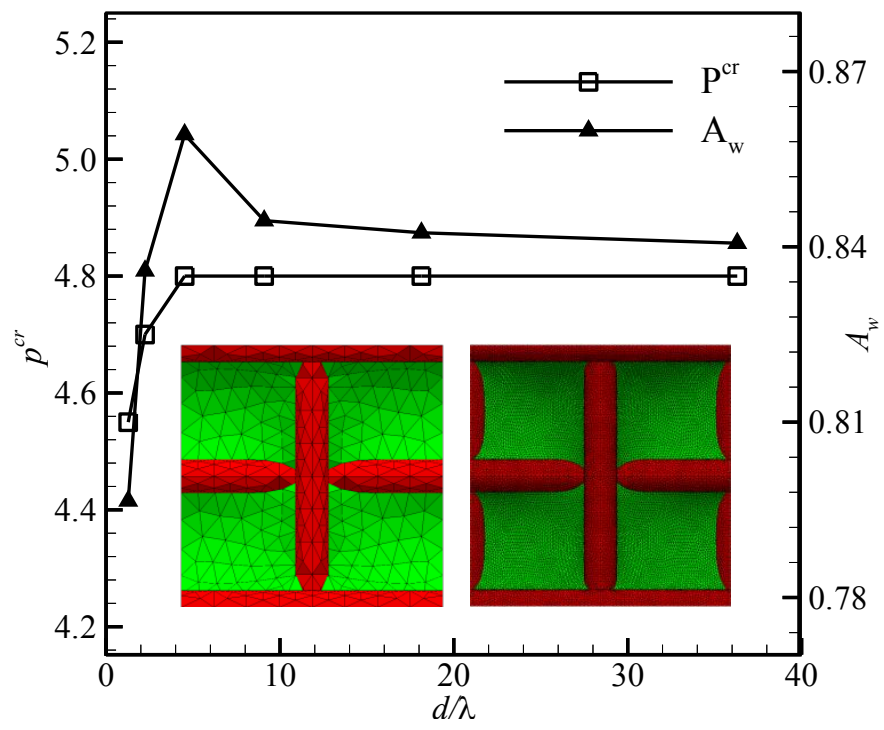

Figure 5 

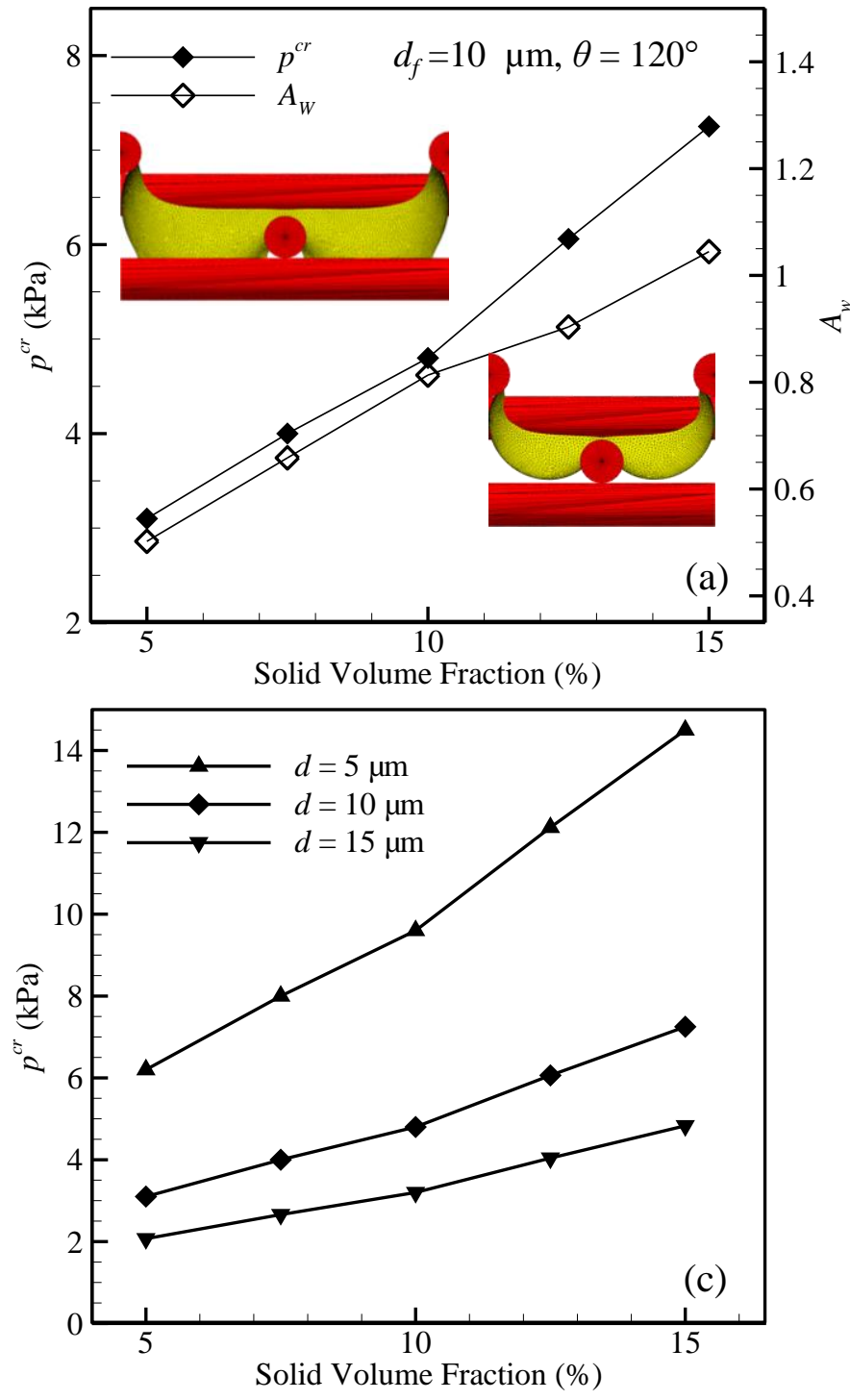

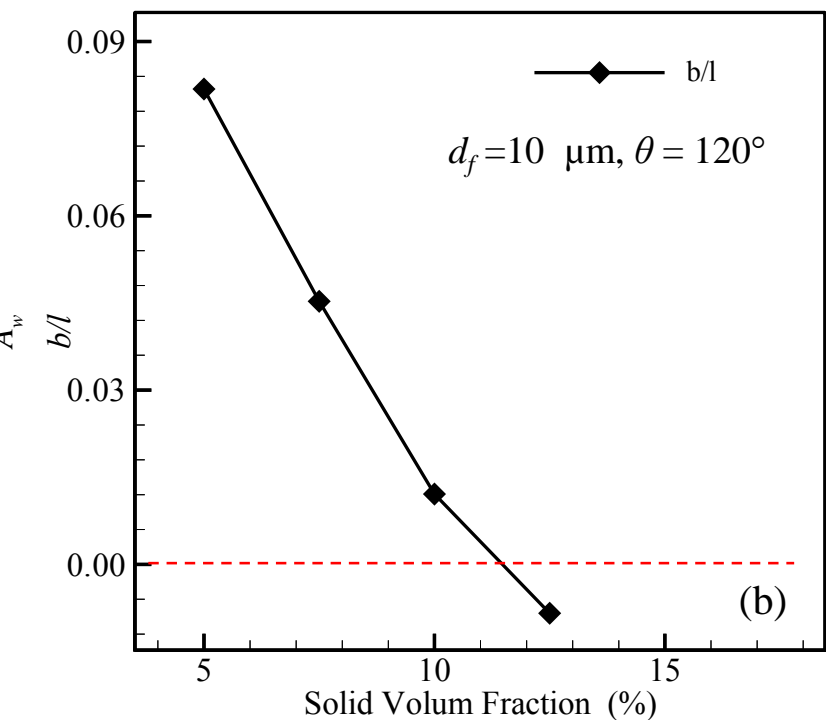

$$
\begin{array}{ll}
p_{F B}^{c r}=1.99 \mathrm{kPa} & p_{F B}^{c r}=4.34 \mathrm{kPa} \\
p_{F M}^{c r}=2.12 \mathrm{kPa} & p_{F M}^{c r}=4.97 \mathrm{kPa} \\
p_{L i t}^{c r}=1.06 \mathrm{kPa} & p_{L i t}^{c r}=2.49 \mathrm{kPa}
\end{array}
$$
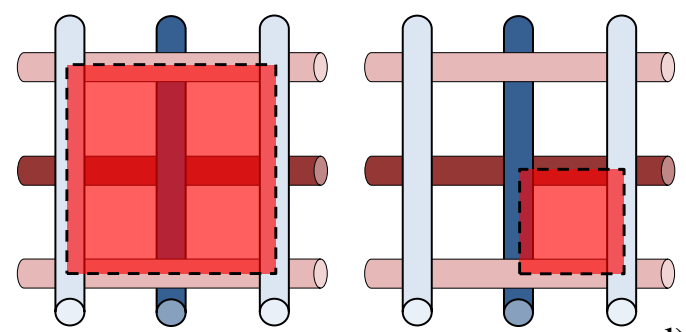

d) 

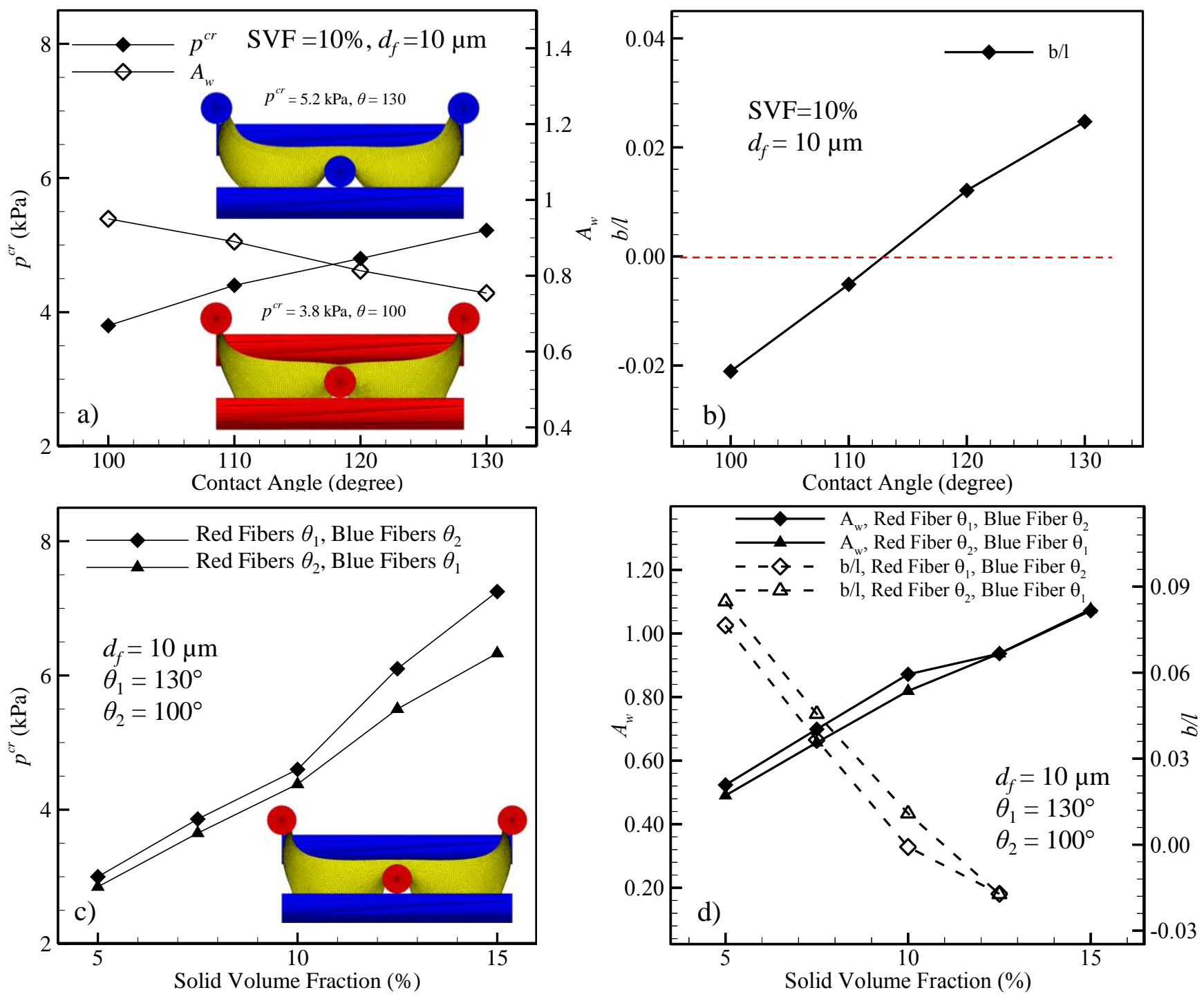


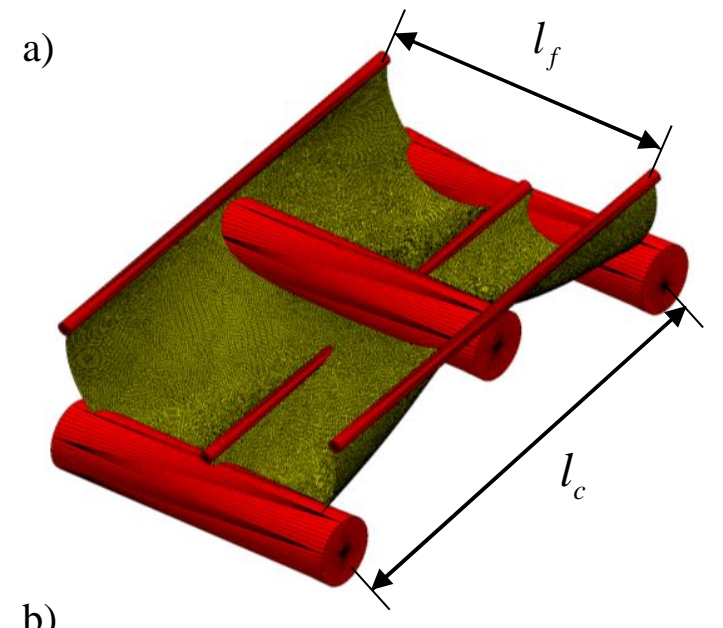

b)

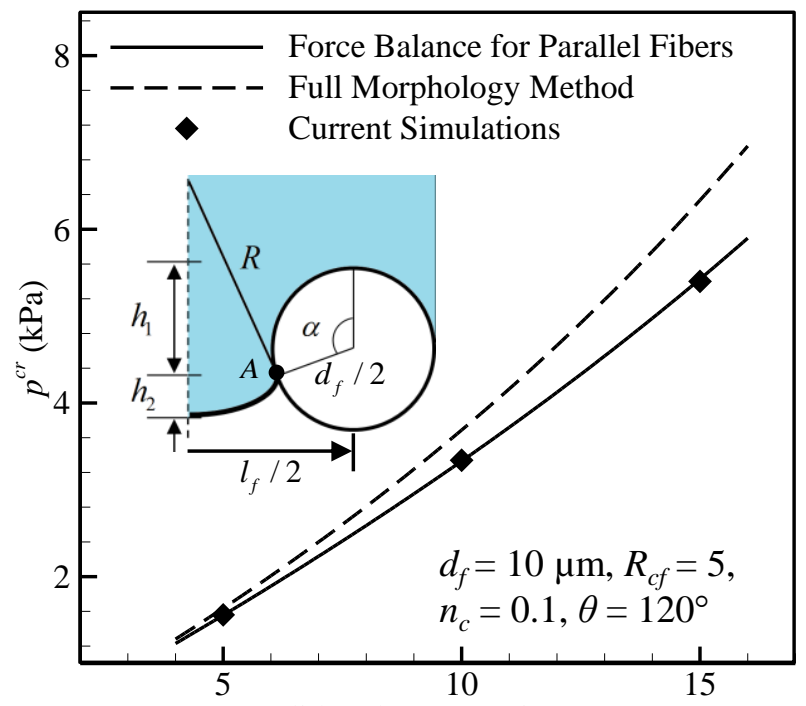

c)

Solid Volume Fraction (\%)

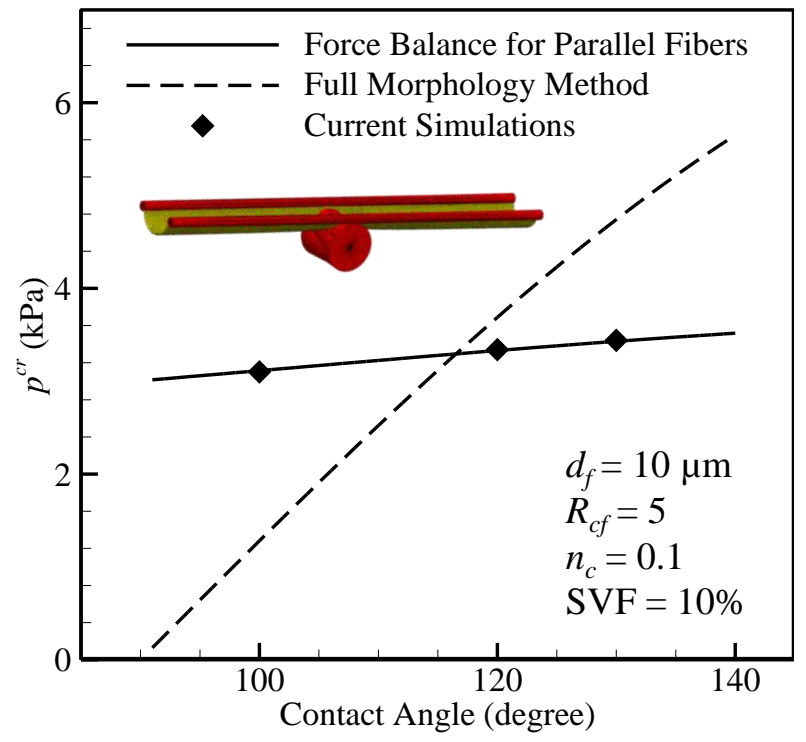

\section{Figure 8}




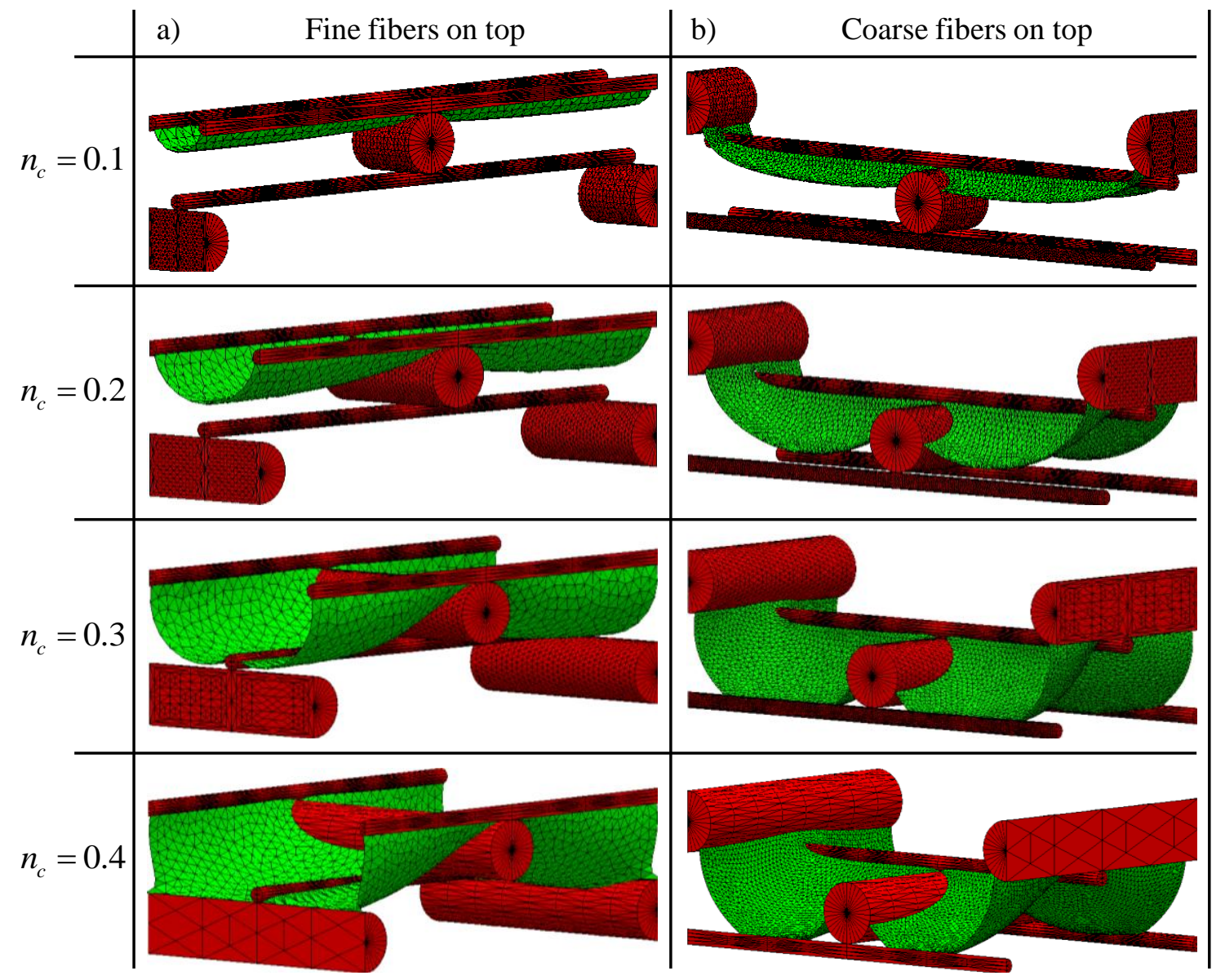

\section{Figure 9}


a)

Fine fibers on top

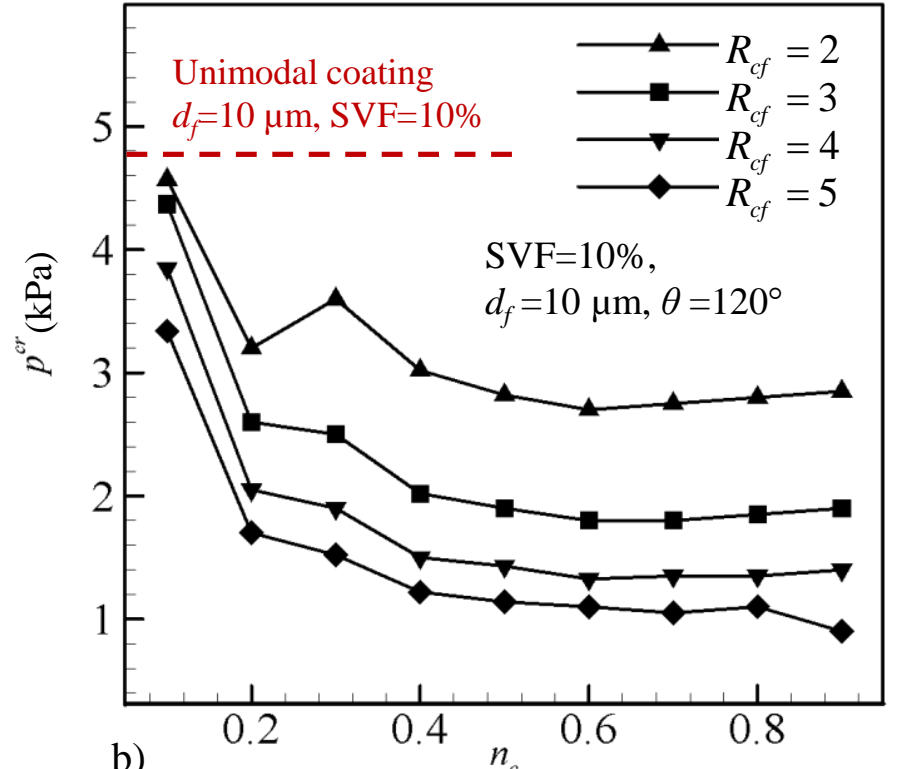

b)

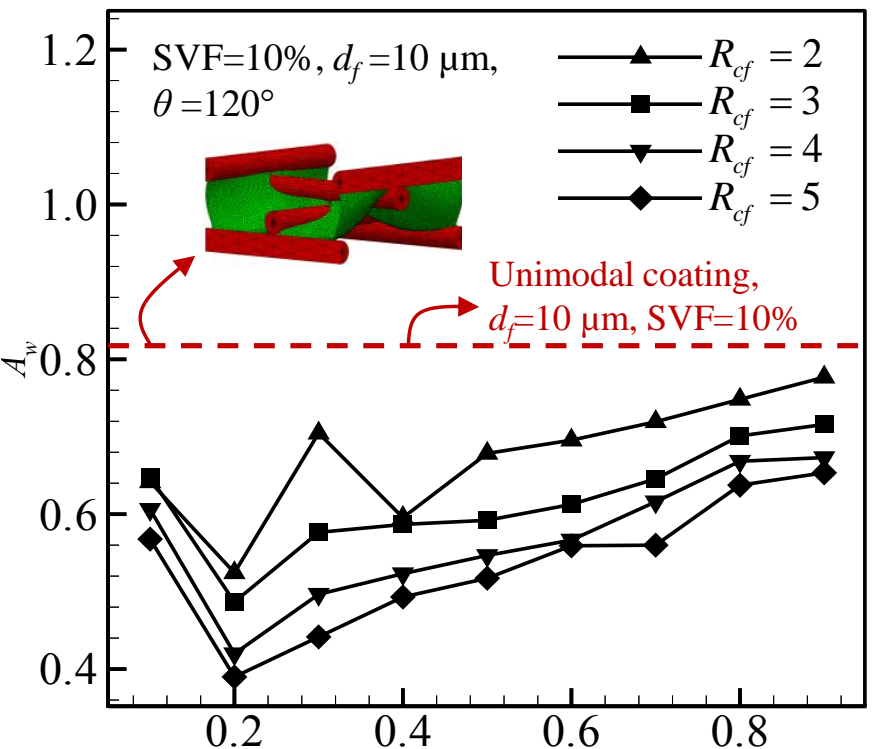

c)

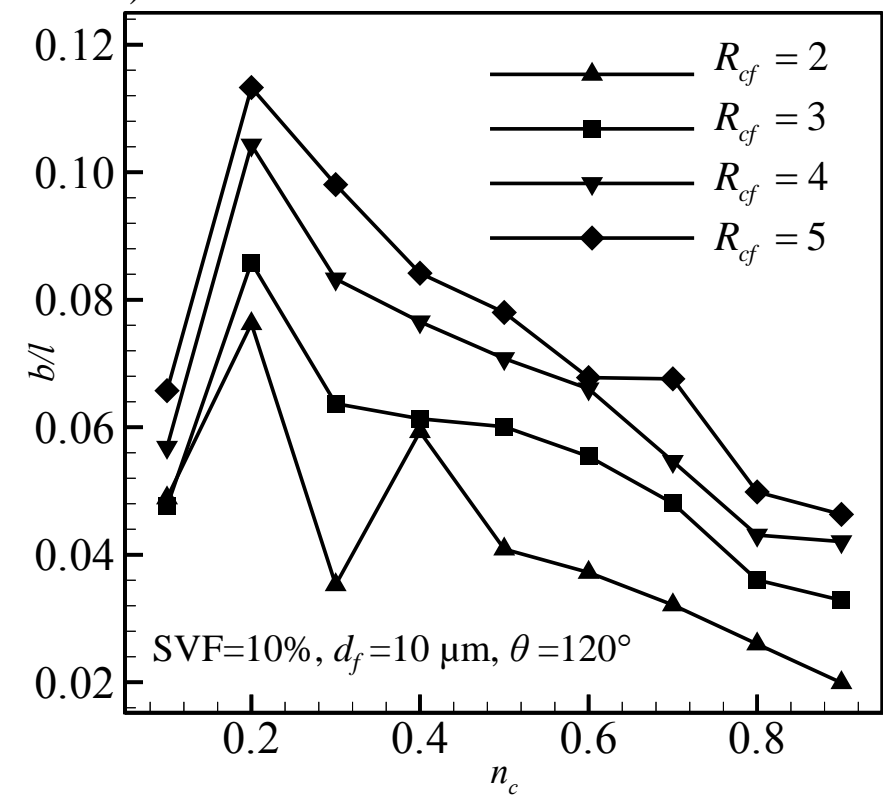

d)

Coarse fibers on top
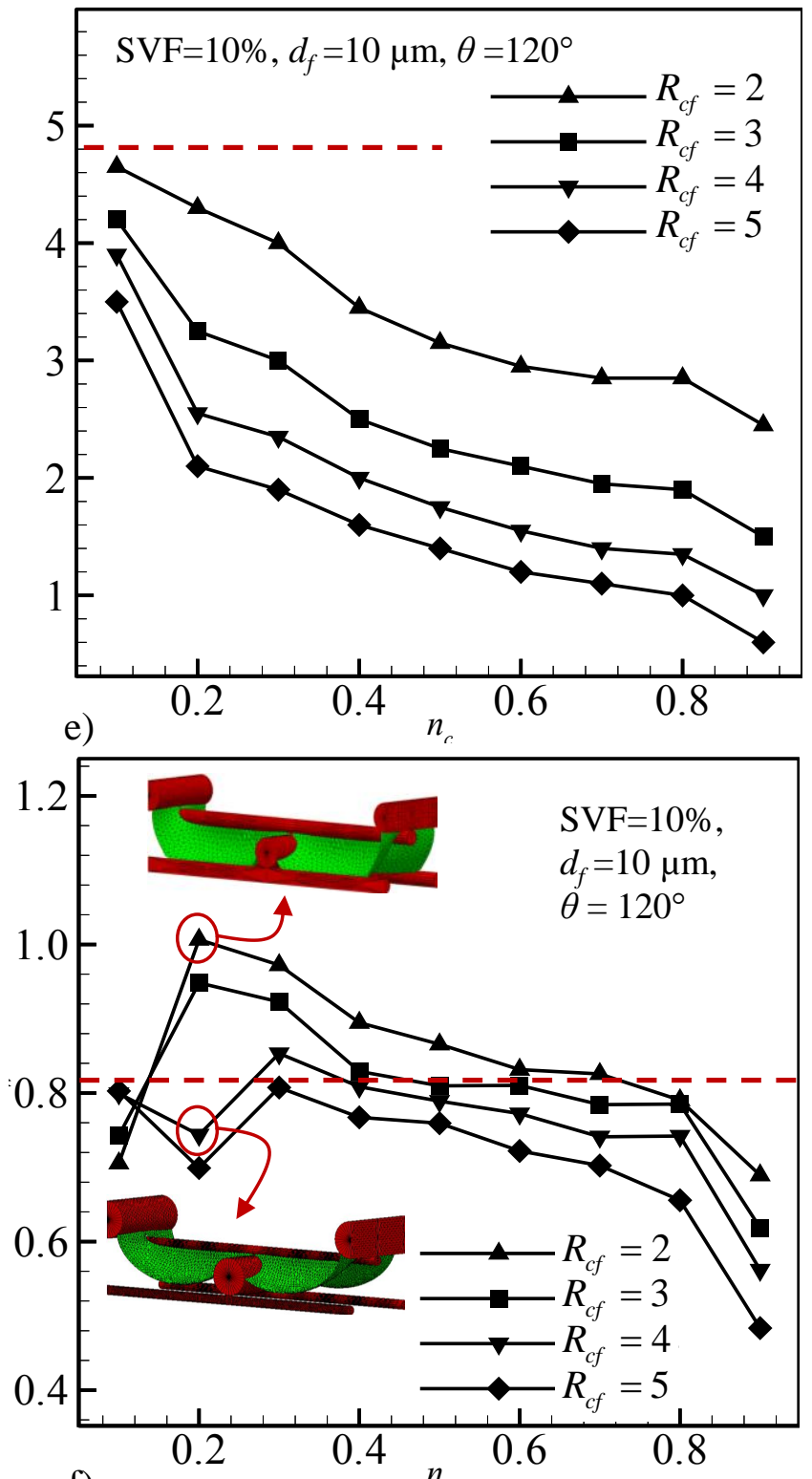

f)

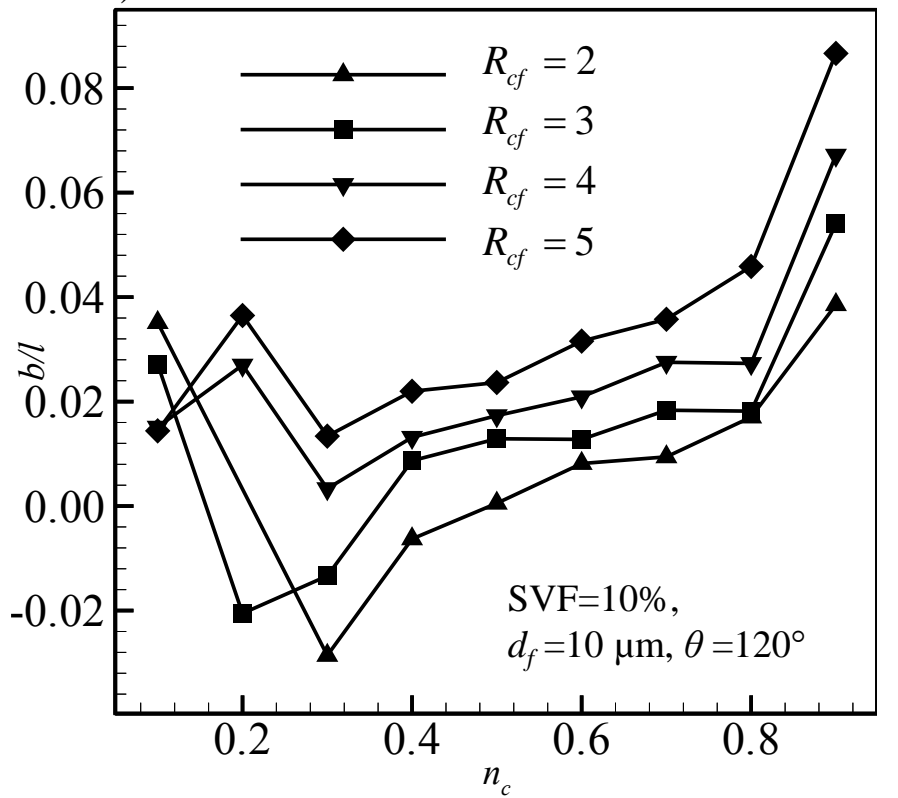

